ORIGINAL ARTICLE

\title{
How well do we investigate patients with suspected subarachnoid haemorrhage? The continuing need for cerebrospinal fluid investigations
}

\author{
M L A Schofield, E Lorenz, T J Hodgson, S Yates, P D Griffiths
}

Postgrad Med J 2004;80:27-30. doi: 10.1136/pgmi.2003.005918

See end of article for authors' affiliations

Correspondence to

Correspondence to:
Professor Paul D Griffiths, Academic Unit of

Radiology, Floor C, Royal

Hallamshire Hospital,

Glossop Road, Sheffield

S10 2JF, UK

P.Griffiths@Sheffield.ac.uk

Submitted 30 January 2003

Accepted 17 May 2003

\begin{abstract}
Objective: To demonstrate the extent of compliance with established guidelines for the investigation of suspected subarachnoid haemorrhage (SAH) and the implications of non-compliance.

Design: Prospective observational study of practice in three hospitals in the Trent region.

Setting: One teaching hospital with a tertiary neuroscience referral centre and two large district general hospitals.

Participants: 50 consecutive patients from each centre referred for suspected SAH with negative computed tomography.

Main outcome measures: Diagnosis of SAH confirmed or excluded according to guidelines.

Results: When the data from the three centres were combined ( $n=150$ computed tomography negative cases) cerebrospinal fluid (CSF) investigation was not performed in 60/150 (40\%). In the 90 cases where CSF studies were performed SAH was confirmed in 11 (12\%).

Conclusion: There is significant non-compliance in following the established guidelines for the investigation of SAH at the centres studied. As the primary cause of non-traumatic SAH is ruptured aneurysm, which is associated with high morbidity and mortality from second haemorrhage, this highlights a major source of concern for clinical governance.
\end{abstract}

$\mathrm{T}$ he majority of patients who present with headache will have non-life threatening conditions such as tension type headache or migraine; however, a small proportion of cases will have potentially serious pathology of which subarachnoid haemorrhage ( $\mathrm{SAH}$ ) is a major cause.

The classical presentation in cases of SAH is sudden onset of headache, frequently described as the worst ever experienced, there may be transient loss of consciousness or focal neurological deficit, and a history of associated vomiting is often given. It is well recognised that there is a spectrum of clinical presentations and many cases will not fit the classical profile. Due to these problems guidelines for the diagnostic pathway have been produced and recommended by the Society of British Neurological Surgeons (Mayberg et al and fig 1). In summary, the guidelines state where clinical presentation suggests SAH, $x$-ray computed tomography of the brain should be performed and when computed tomography is negative or equivocal cerebrospinal fluid (CSF) should be examined.

The incidence of spontaneous SAH in the UK is approximately $7.5-12.6$ per 100000 person-years ${ }^{2}$ and in over $80 \%$ the haemorrhage is due to a ruptured intracranial aneurysm. ${ }^{3}$

If the haemorrhage is due to a ruptured aneurysm and the initial bleed is survived, the risk of re-bleeding is high with attendant has high mortality and morbidity rates. ${ }^{3}$ These can be reduced significantly if the diagnosis is confirmed and intervention by endovascular or conventional neurosurgical techniques takes place.

There are four major reasons for the misdiagnosis of SAH:

1. The spectrum of clinical presentation.

2. The limitations of the sensitivity of cranial computed tomograms; computed tomography is highly sensitive on day 1 with a $92 \%-98 \%$ positivity which falls precipitously five days after ictus to $80 \%$, then to $50 \%$ on day 7 , and to $30 \%$ on day $14 .{ }^{45}$
3. Misinterpretation of computed tomograms.

4. Failure to perform CSF examination after a normal computed tomogram. The sensitivity of CSF investigation remains close to $100 \%$ over 14 days $^{3}$ from ictus.

It should be noted that if the diagnostic pathway is followed, the errors, as a result of factors 2 and 3, are not significant as the CSF investigation will be positive in these cases.

The aim of this study was to assess the level of compliance with the established guidelines in three hospitals, specifically judging how frequently CSF studies were omitted in the face of a negative computed tomography and to estimate the clinical significance of that error.

\section{METHODS}

This was a prospective study at a teaching hospital in South Yorkshire that houses the regional neuroscience centre and two large district general hospitals with 600 and 800 beds respectively. There was consecutive enrolment of patients where cranial computed tomography was requested for investigation of suspected SAH as stated on the request card, recruitment being completed when 50 negative computed tomograms had been collected at each centre. This took six months (June to November 2000) at the teaching hospital, 10 months (September 2001 to June 2002) in district general hospital A, and eight months (January to August 2001) in the district general hospital $\mathrm{B}$.

At the teaching hospital all scans were performed on a General Electric Fxi CT scanner (General Electric, Milwaukee, USA) acquiring $3 \mathrm{~mm}$ slices through the posterior fossa and

Abbreviations: CSF, cerebrospinal fluid; SAH, subarachnoid haemorrhage 


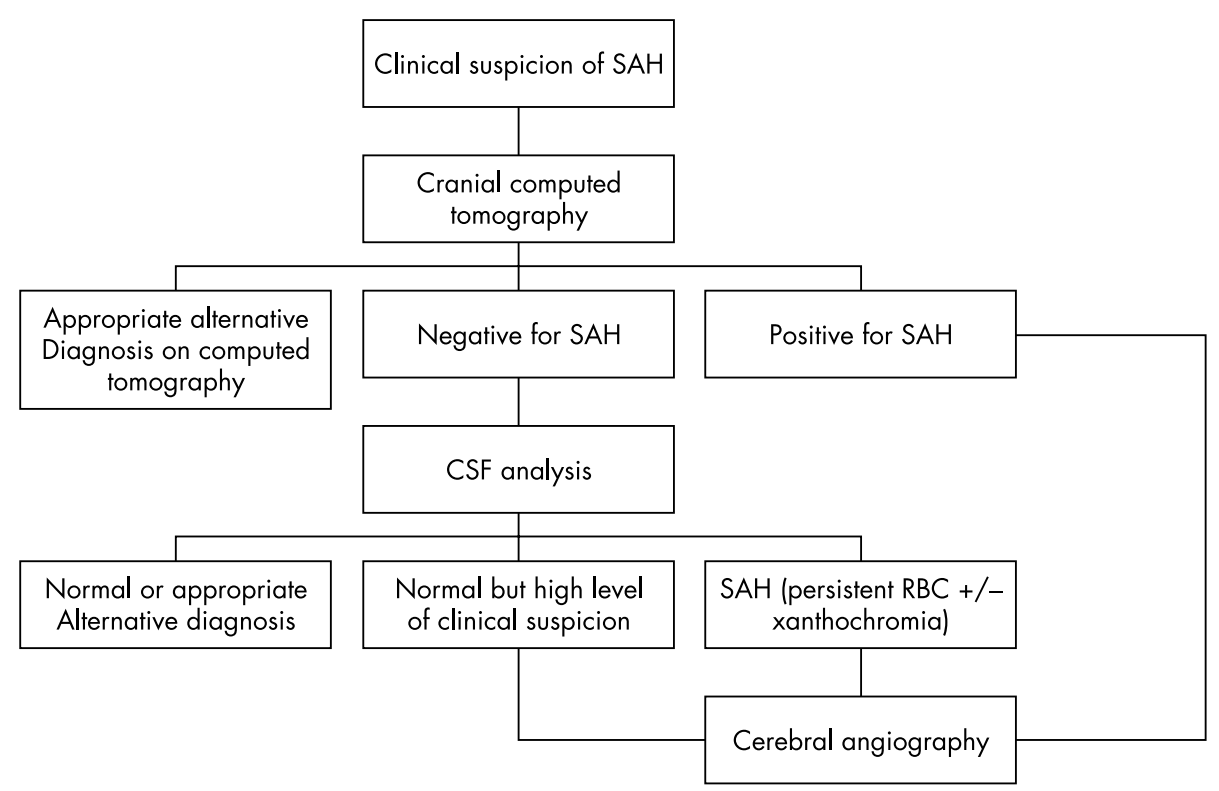

Figure 1 Summary of the Guidelines of the Society of British Neurological Surgeons.

$10 \mathrm{~mm}$ slices to the top of the head. All scans were reported by one of four consultant neuroradiologists. At the district general hospital A, all scans were performed on Marconi Mx 8000 with $5 \mathrm{~mm}$ slices throughout the head and reported by a consultant radiologist. At the district general hospital B, all scans were performed on a Siemens Volume Access multislice computed tomography scanner (Siemens, Erlangen, Germany) with a standard protocol of $5 \mathrm{~mm}$ and $10 \mathrm{~mm}$ slices and reported by a consultant radiologist.

The case notes of all the patients included in the study were reviewed. The information retrieved included demographic data, the result of the computed tomography, and the timing of the scan in relation to onset of ictus, which was categorised into three commonly accepted phases: acute $(<4$ days), subacute (4-14 days), and chronic (>14 days). For those 150 patients with a normal computed tomography further information regarding the lumbar puncture was obtained. This included whether a lumbar puncture was performed, the timing of the lumbar puncture from point of ictus, and the result of CSF examination including the spectrophotometric analysis for xanthochromia where performed. If a lumbar puncture was not performed, the reason for this was identified according to the clinical notes.

The computed tomograms at the district general hospital which were reported as normal but had a positive lumbar puncture were reviewed by a consultant radiologist.

All the patients were then subdivided into the following groups:

- Group 1: computed tomogram positive for pathology which did not require a lumbar puncture including those demonstrating SAH.

- Group 2: computed tomography negative for SAH and lumbar puncture performed; computed tomography negative for SAH and no lumbar puncture performed; computed tomography negative for $\mathrm{SAH}$, refused/failed lumbar puncture.

\section{RESULTS}

Over the period of the study 180 patients were studied in order to have access to 150 computed tomography negative cases. The 29 patients in group 1 (positive for pathology obviating the need for lumbar puncture) included: SAH $(n=13)$, intraparenchymal haemorrhage $(n=12)$, giant unruptured posterior communicating artery aneurysm $(\mathrm{n}=1)$, and cerebral oedema due to meningococcal septicaemia/meningitis $(\mathrm{n}=1)$.

The 150 cases in group 2 (computed tomogram negative) consisted of 64 males and 86 females with a mean age of 42 years (range $17-88$ years).

The results of their further clinical investigations are shown in table 1 . This shows that in $40 \%$ of cases the patient with a negative computed tomogram did not have a CSF investigation. The figures were slightly lower at the teaching hospital (32\%) when compared with both district general hospitals $(44 \%)$.

CSF studies were done in 90/150 (60\%) of patients and in $11 / 90(12 \%)$ CSF analysis confirmed SAH. Eight were imaged in the acute phase, two in the subacute phase, and one in the chronic phase.

In the majority of cases no valid reason was identified from the case notes for omission of CSF analysis. However a couple of interesting patterns were noted: included in group 2 are six cases with evidence of cerebral infarction on computed tomography. On review by a consultant radiologist, two were in keeping with a recent infarct and one computed tomogram was normal. Also, in eight cases it was deemed unnecessary to perform a lumbar puncture as there had been resolution of symptoms by the next morning. At one district general hospital, in one of the cases, the scan was reported as normal but the lumbar puncture was positive and on review the computed tomogram showed subarachnoid blood in the sylvian fissure and the interhemisheric fissure (figs 2 and 3 ). A cerebral angiogram demonstrated the aneurysm that was successfully embolised.

\section{DISCUSSION}

The guidelines for the investigation of SAH are well established but we have demonstrated a significant level of non-compliance in both a teaching hospital with a neuroscience centre and two large district general hospitals. In our cases, $12 \%$ of patients are diagnosed with SAH after lumbar puncture despite normal computed tomography. It is 
Table 1 Summary of the frequency of not performing CSF studies in 150 patients (50 from three hospitals) with normal computed tomograms being performed for the investigation of possible subarachnoid haemorrhage (percentages in parentheses)

\begin{tabular}{|c|c|c|c|c|c|}
\hline & \multirow{2}{*}{$\begin{array}{l}\text { CSF study not } \\
\text { performed }\end{array}$} & \multicolumn{2}{|c|}{ Recorded reason for no CSF study } & \multicolumn{2}{|c|}{ Result of CSF study when performed } \\
\hline & & No valid reason & Failed or refused & Normal & SAH \\
\hline Teaching hospital & $16 / 50(32)$ & $12 / 16$ & $4 / 16$ & $31 / 34$ & $3 / 34(9)$ \\
\hline District general hospital A & $22 / 50(44)$ & $18 / 22$ & $4 / 22$ & $26 / 28$ & $2 / 28(7)$ \\
\hline District general hospital B & $22 / 50(44)$ & $20 / 22$ & $2 / 22$ & $22 / 28$ & $6 / 28(21)$ \\
\hline Total & $60 / 150(40)$ & $50 / 60$ & $10 / 60$ & $79 / 90$ & $11 / 90(12)$ \\
\hline
\end{tabular}

reasonable to postulate that there is potentially a similar proportion of patients who are inadequately investigated and discharged but have the potential for another perhaps fatal bleed.

\section{Why are the guidelines not followed?}

Although computed tomography is most sensitive within 24 hours of ictus, this is still not an absolute reference standard as $2 \%-8 \%$ of SAH will not be visible. The precipitous fall-off in sensitivity after five days may not be recognised by some clinicians and so if CSF analysis is not used as a safety net there will be a greater number of "missed" cases. Interestingly, in our study the majority of patients who had SAH confirmed on CSF analysis despite normal computed tomography were investigated in the acute phase. This must raise the level of concern for the proportion of "missed" cases when the imaging is performed in the subacute or chronic phase with no subsequent CSF examination.
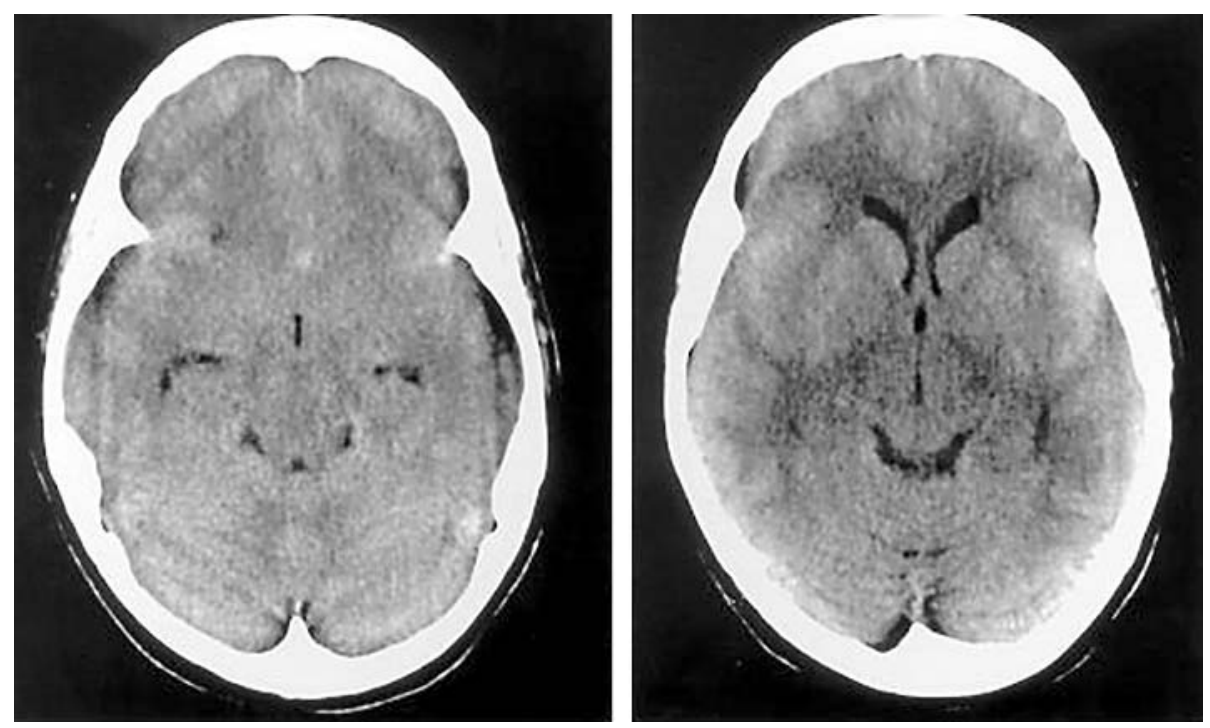

Figure 2 Computed tomogram of head at the level of the temporal horns (left) and foramina of Monro (right) demonstrating subarachnoid blood in the sylvian and interhemispheric fissures. This scan was reported as normal at a district general hospital and the lumbar puncture was positive.
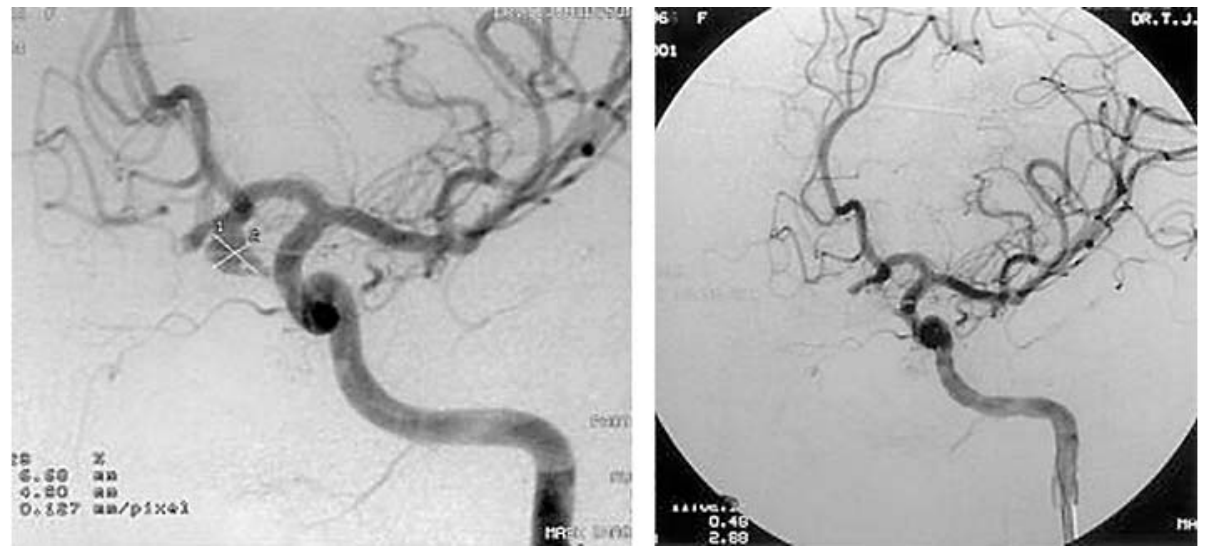

Figure 3 In the same patient as in fig 2, the cerebral angiogram demonstrates the aneurysm before treatment (left) and after successful embolisation (right). 
The importance of CSF analysis should not be underestimated. Xanthochromia detected either visually or by spectrophotometry, where available, avoids the pitfall of the traumatic tap. ${ }^{6}$ It becomes detectable between six and 12 hours after ictus. ${ }^{7}$ Although, it is preferable to delay the lumbar puncture until 12 hours after ictus to avoid the possibility of a false negative result in the detection of CSF xanthochromia.

If CSF undergoes prompt comprehensive analysis including spectrophotometry it maintains a sensitivity of $100 \%$ in the detection of SAH for up to 14 days after ictus falling to $70 \%$ after three weeks and $40 \%$ by four weeks. ${ }^{3}$

In our study it was apparent that in some cases CSF analysis was not performed after a normal computed tomogram as there had been resolution of symptoms. Unfortunately this is a recognised occurrence in SAH and the headache may resolve spontaneously or with simple analgesia.

In six cases there was evidence of cerebral infarction on computed tomography. This may have been assumed to be thromboembolic by the clinicians but it must be remembered that arterial spasm leading to ischaemia is a common sequel of SAH.

The guidelines for the investigation of SAH are well established but we have confirmed that the level of noncompliance is high and nearly as prevalent in the teaching hospital as in the district general hospital. We suspect that the problem is likely to be nationwide on the basis of an increasing number of litigation cases seen by the senior authors over the last few years. We believe that there is urgent need for the profile of the guidelines to be raised and more education as to the shortcomings of computed tomography in cases of suspected SAH.

\section{Authors' affiliations}

M L A Schofield, E Lorenz, T J Hodgson, Department of Radiology, Royal Hallamshire Hospital, Sheffield, UK

$S$ Yates, Barnsley District Hospital, Barnsley, UK

P D Griffiths, Academic Unit of Radiology, University of Sheffield, Sheffield, UK

\section{REFERENCES}

1 Mayberg MR, Batjer HH, Dacey R, et al. Guidelines for the management of aneurysmal subarachnoid haemorrhage. Stroke 1994;25:2315-28.

2 Pobereskin LH. Incidence and outcome of subarachnoid haemorrhage: a retrospective population based study. J Neurol Neurosurg Psychiatry 2001;70:340-3.

3 Vermeulen M, van Gijn J. The diagnosis of subarachnoid haemorrhage. J Neurol Neurosurg Psychiatry 1990;53:365-72.

4 Van der Wee N, Rinkel GJE, Hasan D, et al. Detection of subarachnoid haemorrhage on early CT: is lumbar puncture still needed after a negative scan? J Neurol Neurosurg Psychiatry 1995:58:357-9.

5 Kassell NF, Torner JC, Haley EC, et al. The International Cooperative Aneurysm Study on the Timing of Aneurysm Surgery. J Neurosurg 1990;73:18-36.

6 Sengupta RP, McAllister VL. Subarachnoid haemorrhage. Berlin: SpringerVerlag, 1986.

7 Wasserberg J, Barlow P. Lumbar puncture still has an important role in diagnosing SAH. BMJ 1997;315:1598-9. 